ACCEPted for publication in AJ

Preprint typeset using $\mathrm{L}^{A} \mathrm{~T}_{\mathrm{E}} \mathrm{X}$ style emulateapj v. 08/22/09

\title{
DYNAMICAL AND OBSERVATIONAL CONSTRAINTS ON ADDITIONAL PLANETS IN HIGHLY ECCENTRIC PLANETARY SYSTEMS ${ }^{1}$
}

\author{
Robert A. Wittenmyer, Michael Endl, William D. Cochran \\ McDonald Observatory, University of Texas at Austin, Austin, TX 78712 \\ HAROLD F. LEVISON \\ Department of Space Studies, Southwest Research Institute, Boulder, CO 80302 \\ Accepted for publication in AJ
}

\begin{abstract}
Long time coverage and high radial velocity precision have allowed for the discovery of additional objects in known planetary systems. Many of the extrasolar planets detected have highly eccentric orbits, which raises the question of how likely those systems are to host additional planets. We investigate six systems which contain a very eccentric $(e>0.6)$ planet: HD 3651, HD 37605, HD 45350, HD 80606, HD 89744, and 16 Cyg B. We present updated radial-velocity observations and orbital solutions, search for additional planets, and perform test particle simulations to find regions of dynamical stability. The dynamical simulations show that short-period planets could exist in the HD 45350 and 16 Cyg B systems, and we use the observational data to set tight detection limits, which rule out additional planets down to a few Neptune masses in the HD 3651, HD 45350, and 16 Cyg B systems. Subject headings: extrasolar planets - planetary dynamics - stars: planetary systems
\end{abstract}

\section{INTRODUCTION}

One surprising result that has come out of the more than 200 extrasolar planet discoveries to date is the wide range of eccentricities observed. Unlike our own Solar system, many of the extrasolar planets which are not tidally locked to their host stars have moderate eccentricities $(e>0.2)$, and 15 planets have high eccentricities $(e>0.6)$. These observations have spawned several theories as to the origin of highly eccentric extrasolar planets. One such method, planet-planet scattering, occurs when multiple jovian planets form several astronomical units (AU) from the host star and then interact, leaving one in an eccentric orbit and often ejecting the other (Rasio \& Ford 1996). This method has been proposed to explain the architecture of the $v$ And planetary system (Ford et al. 2005), which contains a hot Jupiter as well as two jovian planets in moderately eccentric orbits. Lin \& Ida (1997) suggested a merger scenario in which inner protoplanets perturb each other and merge to form a single massive, eccentric planet with $e \gtrsim 0.3$ and $a \sim 0.5-1$ AU.

Interactions with stellar companions are another possible way to boost a planet's eccentricity. Of the 15 stars hosting a planet with $e>0.6$, six are also known to possess stellar-mass companions in wide binary orbits: HD 3651 (Mugrauer et al. 2006; Luhman et al. 2007), HD 20782 (Desidera \& Barbieri 2007), HD 80606, HD 89744 (Wilson et al. 2001; Mugrauer et al. 2004), 16 Cyg B, and HD 222582 (Raghavan et al. 2006). If the inclination angle between the planetary orbit and a stellar companion is large, the Kozai mechanism Kozai

\footnotetext{
Electronic address: robw@astro.as.utexas.edu

1 Based on observations obtained with the Hobby-Eberly Telescope, which is a joint project of the University of Texas at Austin, the Pennsylvania State University, Stanford University, LudwigMaximilians-Universität München, and Georg-August-Universität Göttingen.
}

1962) can induce large-amplitude oscillations in the eccentricity of the planet (e.g. Malmberg et al. 2006). These oscillations can be damped by general relativistic effects and by interaction with other planets, and hence are most effective in systems with a single planet in an orbit $a \gtrsim 1 \mathrm{AU}$ from the host star (Takeda \& Rasio 2005). The Kozai mechanism has been suggested to explain the high eccentricity of $16 \mathrm{Cyg} \mathrm{Bb}$ (Holman et al. 1997; Mazeh et al. 1997) and HD 80606b (Wu \& Murray 2003). Hauser \& Marcy (1999) found the inclination of 16 Cyg B orbiting the system barycenter to lie between 100 and 160 degrees, where 90 degrees is an edge-on orientation. However, it is the difference in inclination between the orbital planes of the planetary and stellar companion that is critical in determining the importance of the Kozai mechanism, and the inclination of the planet's orbit is generally not known for non-transiting systems.

Of the 192 known planetary systems, $23 \quad(12 \%)$ are multi-planet systems. Recent discoveries of additional objects in systems known to host at least one planet (Udrv et al. 2007; Wittenmver et al. 2007; Rivera et al. 2005; Vogt et al. 2005; McArthur et al. 2004; Santos et al. 2004) suggest that multiple-planet systems are common. Of particular interest are systems which host a jovian planet and a low-mass "hot Neptune," e.g. 55 Cnc (=HD 75732), GJ 876, $\mu$ Arae (=HD 160691), Gl 777A (=HD 190360). Motivated by the discoveries of hot Neptunes in known planetary systems, we have undertaken an intensive survey of selected single-planet systems to search for additional low-mass companions. Three of the planetary systems discussed in this paper (HD 3651, HD 80606, HD 89744) are part of this campaign. The excellent radial-velocity precision of the High Resolution Spectrograph on the Hobby-Eberly Telescope (HET), combined with queue-scheduling, allow us to time the observations in such a way as to minimize phase gaps in the orbit of the known planet, and 
also to act quickly on potential new planet candidates. The use of the HET in this manner is discussed further in Cochran et al. (2004) with regard to the discovery of HD 37605 b.

In this work, we aim to combine observational limits on additional planets in known planetary systems with dynamical constraints obtained by N-body simulations. The observations address the question: What additional planets are (or are not) in these systems? The dynamical simulations can answer the question: Where are additional planets possible? Section 2 describes the observations and the test particle simulations for six highly eccentric planetary systems: HD 3651, HD 37605, HD 45350, HD 80606, HD 89744, and 16 Cyg B. We have chosen these systems based on two criteria: (1) Each hosts a planet with $e>0.6$, and (2) Each has been observed by the planet search programs at McDonald Observatory. In $\S 3$, we present and discuss the results of the updated orbital fits, dynamical simulations, and detection limit computations.

\section{OBSERVATIONS AND DATA ANALYSIS}

\subsection{Radial-Velocity Observations}

Five of the six stars considered in this work have been observed with the McDonald Observatory $9.2 \mathrm{~m}$ Hobby-Eberly Telescope (HET) using its High Resolution Spectrograph (HRS) (Tull 1998). A full description of the HET planet search program is given in Cochran et al. (2004). For 16 Cyg B, observations from McDonald Observatory were obtained only with the $2.7 \mathrm{~m}$ Harlan J. Smith (HJS) telescope; the long-term planet search program on this telescope is described in Wittenmyer et al. (2006). All available published data on these systems were combined with our data from McDonald Observatory in the orbit fitting procedures.

\subsection{Numerical Methods}

To place constraints on the architecture of planetary systems, we would like to know where additional objects can remain in stable orbits in the presence of the known planet(s). We performed test particle simulations using SWIFT ${ }^{2}$ (Levison \& Duncan 1994) to investigate the dynamical possibility of additional low-mass planets in each of the six systems considered here. Low-mass planets can be treated as test particles since the exchange of angular momentum with jovian planets is small. We chose the regularized mixed-variable symplectic integrator (RMVS3) version of SWIFT for its ability to handle close approaches between massless, non-interacting test particles and planets. Particles are removed if they are (1) closer than 1 Hill radius to the planet, (2) closer than $0.05 \mathrm{AU}$ to the star, or (3) farther than $10 \mathrm{AU}$ from the star. Since the purpose of these simulations is to determine the regions in which additional planets could remain in stable orbits, we set this outer boundary because the current repository of radial-velocity data cannot detect objects at such distances.

The test particle simulations were set up following the methods used in Barnes \& Raymond (2004), with the exception that only initially circular orbits are consid-

2 SWIFT is publicly available at http://www.boulder.swri.edu/ hal/swift.html ered in this work. For each planetary system, test particles were placed in initially circular orbits spaced every 0.002 AU in the region between 0.05-2.0 AU. We have chosen to focus on this region because the duration of our high-precision HET data is currently only 2-4 years for the objects in this study. The test particles were coplanar with the existing planet, which had the effect of confining the simulation to two dimensions. Input physical parameters for the known planet in each system were obtained from our Keplerian orbit fits described in $\S 3.1$, and from recent literature for 16 Cyg B (Wittenmyer et al. 2007) and HD 45350 (Endl et al. 2006). The planetary masses were taken to be their minimum values $(\sin i=1)$. The systems were integrated for $10^{7} \mathrm{yr}$, following Barnes \& Ravmond (2004) and allowing completion of the computations in a reasonable time. We observed that nearly all of the test-particle removals occurred within the first $10^{6} \mathrm{yr}$; after this time, the simulations had essentially stabilized to their final configurations.

\section{RESULTS AND DISCUSSION}

\subsection{Updated Keplerian Solutions for 4 Systems}

We present updated Keplerian orbital solutions for HD 3651b, HD 37605b, HD 80606b, and HD 89744b in Table 1. A summary of the data used in our analysis is given in Table 2, and the HET radial velocities are given in Tables 3-6. The velocity uncertainties given for the HET data represent internal errors only, and do not include any external sources of error such as stellar "jitter." The parameters for the remaining two planets, HD 45350b and $16 \mathrm{Cyg} \mathrm{Bb}$, are taken from Endl et al. (2006) andWittenmyer et al. (2007), respectively. Radial velocity measurements from the HET are given for HD 45350 in Endl et al. (2006), and velocities for $16 \mathrm{Cyg} \mathrm{B}$ from the HJS telescope are given in Wittenmyer et al. (2007). As in Wittenmyer et al. (2007), all available published data were combined with those from McDonald, and the known planet in each system was fit with a Keplerian orbit using GaussFit (Jefferys et al. 1987), allowing the velocity offset between each data set to be a free parameter. Examination of the residuals to our Keplerian orbit fits revealed no evidence for additional objects in any of the six systems in this study.

The Saturn-mass ( $\mathrm{M} \sin i=0.2 \mathrm{M}_{\mathrm{Jup}}$ ) planet HD $3651 \mathrm{~b}$ was discovered by Fischer et al. (2003) using observations from Lick and Keck. We fit these data, which were updated in Butler et al. (2006), in combination with observations from the HJS and HET at McDonald Observatory. The HET data, which consist of multiple exposures per visit, were binned using the inverse-variance weighted mean value of the velocities in each visit. The standard error of the mean was added in quadrature to the weighted rms about the mean velocity to generate the error bar of each binned point $(\mathrm{N}=29)$. The rms about the combined fit for each dataset is: Lick \& Keck$6.6 \mathrm{~m} \mathrm{~s}^{-1}$, HET-9.4 $\mathrm{m} \mathrm{s}^{-1}$, HJS-12.2 $\mathrm{m} \mathrm{s}^{-1}$. The fitted orbital parameters for HD 3651b are of comparable precision to those reported in Butler et al. (2006), and agree within $2 \sigma$. The recent discovery of a $\mathrm{T}$ dwarf companion to HD 3651 (Mugrauer et al. 2006; Luhman et al. 2007) prompts an interesting exercise: Can the radial-velocity 
trend due to this object be detected in the residuals after removing the planet? We detect a slope of $-0.27 \pm 0.05$ $\mathrm{m} \mathrm{s}^{-1} \mathrm{yr}^{-1}$, indicating that we are indeed able to discern a trend which is possibly due to the binary companion. However, the reduced $\chi^{2}$ of the orbital solution is not significantly improved by the inclusion of a linear trend $\left(\Delta \chi_{\nu}^{2}=0.18\right)$. The parameters given in Table 1 were obtained from the fit which did not include a trend.

We present 23 new HET observations for HD 37605 obtained since its announcement by Cochran et al. (2004). The data now span a total of 1065 days. The best fit is obtained by including an acceleration of $-20.5 \pm 2.1$ $\mathrm{m} \mathrm{s}^{-1} \mathrm{yr}^{-1}$, indicating a distant orbiting body. Such a finding would lend support to the hypothesis that very eccentric single-planet systems originate by interactions within a wide binary system. The shortest period that this outer companion could have and still remain consistent with the observed acceleration and its uncertainty over the timespan of the observations is about $40 \mathrm{yr}$, assuming a circular orbit. This object would then have a minimum mass in the brown dwarf range.

The planet orbiting HD 80606, first announced by Naef et al. (2001), is the most eccentric extrasolar planet known, with $e=0.933 \pm 0.001$ (Table 1). We have fit the CORALIE data in combination with the Keck data given in Butler et al. (2006) and 23 observations from HET. The extreme velocity variations induced by this planet greatly increase the sensitivity of orbit fits to the weighting of individual measurements. Since the uncertainties of the HET velocities given in Tables 3-6 represent internal errors only, we experimented with adding 1-7 $\mathrm{m} \mathrm{s}^{-1}$ of radial-velocity "jitter" in quadrature before fitting the data for HD 80606. For all of these jitter values, the fitted parameters remained the same within their uncertainties. Table 1 gives the parameters derived from a fit which added $3.5 \mathrm{~m} \mathrm{~s}^{-1}$ of jitter (Butler et al. 2006) to the HET data. The rms about the combined fit is: CORALIE-18.7 $\mathrm{m} \mathrm{s}^{-1}$, HET-7.5 m s${ }^{-1}$, Keck-5.6 $\mathrm{m} \mathrm{s}^{-1}$. Butler et al. (2006) noted that the eccentricity $e$ and the argument of periastron $\omega$ had to be held fixed in their fit to the Keck data alone. However, the large number of measurements included in this work allowed GaussFit to converge with all parameters free.

For HD 89744b, we combine data from the HET with 6 measurements from the HJS telescope and Lick data from Butler et al. (2006). The HET data were binned in the same manner as for $\mathrm{HD} 3651$, resulting in $\mathrm{N}=33$ independent visits. The rms about the combined fit for each dataset is: Lick-17.1 $\mathrm{m} \mathrm{s}^{-1}$, HET-10.7 $\mathrm{m} \mathrm{s}^{-1}$, HJS$9.5 \mathrm{~m} \mathrm{~s}^{-1}$. As with HD 3651b, our derived parameters agree with those of Butler et al. (2006) within 2 $\sigma$. The scatter about our fit remains large, most likely due to the star's early spectral type (F7V), which hinders precision radial-velocity measurements due to the smaller number of spectral lines. For example, the F7V star HD 221287 was recently found to host a planet (Naef et al. 2007); despite the superb instrumental precision of the HARPS spectrograph, that orbital solution has a residual $\mathrm{rms}$ of $8.5 \mathrm{~m} \mathrm{~s}^{-1}$

\subsection{Test Particle Simulations}

The results of the dynamical simulations are shown in Figures 1-3. The survival time of the test particles is plotted against their initial semimajor axis. As shown in Fig- ure 1, the short-period planets HD 3651 and HD 37605 sweep clean the region inside of about $0.5 \mathrm{AU}$. In both of these systems, however, a small number of test particles remained in low-eccentricity orbits near the known planet's apastron distance, near the 1:2 mean-motion resonance (MMR). In the HD 3651 system, particles remained stable beyond about 0.6 AU, which is not surprising given the low mass of the planet. For HD 37605, two distinct strips of stability are seen in Fig. 1, corresponding to the 1:2 and 1:3 MMRs. The eccentricity of the test particles in the region of the 1:2 MMR oscillated between 0.00 and 0.06. Particles in 1:3 MMR oscillated in eccentricity with a larger range, up to $e \sim 0.4$, which is expected due to secular forcing. As with HD 3651, the region beyond about $0.8 \mathrm{AU}$ was essentially unaffected by the planet.

Figure 2 shows the results for the HD 45350 and HD 80606 systems. The long period (963.6 days) and relatively large mass ( $\mathrm{M} \sin i=1.8 \mathrm{M}_{\mathrm{Jup}}$ ) of $\mathrm{HD} 45350 \mathrm{~b}$ restricted stable orbits to the innermost $0.2 \mathrm{AU}$. These test particles oscillated in eccentricity up to $e \sim 0.25$. The $4 \mathrm{M}_{\text {Jup }}$ planet orbiting HD 80606 removed all test particles to a distance of about $1.5 \mathrm{AU}$, and only beyond $1.75 \mathrm{AU}$ did test particles remain in stable orbits for the duration of the simulation $\left(10^{7} \mathrm{yr}\right)$. A region of instability is evident at $1.9 \mathrm{AU}$ due to the $8: 1 \mathrm{MMR}$. Figure 3 shows that HD 89744 b eliminated all test particles except for a narrow region near the 8:3 resonance. For the 16 Cyg B system, only particles inside of about $0.3 \mathrm{AU}$ remained stable, leaving open the possibility of short-period planets. The surviving particles oscillated in eccentricity up to $e \sim 0.45$, but these simulations treat the star as a point mass, and hence tidal damping of the eccentricity is not included. Our results are consistent with those of Menou \& Tabachnik (2003), who investigated dynamical stability in extrasolar planetary systems and found that no test particles survived in the habitable zones of the HD 80606, HD 89744, and 16 Cyg B systems.

\subsection{Detection Limits}

Three of these systems (HD 3651, HD 80606, HD 89744) were monitored intensely with the HET as part of a larger effort to search for low-mass, short period planets. No evidence was found for any such objects in these or any of the six systems in this work. We then asked what limits can be set on additional planets using the high-precision HET data we have obtained. The procedure for determining companion limits was identical to the method described in Wittenmyer et al. (2006), except that in this work, the best-fit Keplerian orbit for the known planet (see $\S 3.1$ ) was removed before performing the limits computations. In this way, we determined the radial-velocity amplitude $K$ for which $99 \%$ of planets would have been detected in the residuals. The eccentricity of the injected test signals was chosen to be the mean eccentricity of the surviving particles from the simulations described in $\S 3.2$. Only the regions in which test particles survived were considered in these limits computations.

The results of these computations were highly varied, reflecting the differing observing strategies employed for these six objects. In particular, HD 3651, HD 80606, and HD 89744 were monitored intensely with the HET as part of a search for short-period objects, whereas 
HD 37605 and HD 45350 were only observed sporadically after the known planet orbits were defined and published (Cochran et al. 2004; Endl et al. 2006), and 16 Cyg B has only been observed with the HJS telescope at a frequency of at most once per month. The companion limits are shown in Figures 4-6; planets with masses above the solid line can be ruled out by the data with $99 \%$ confidence. Not surprisingly, the tightest limits were obtained for HD 3651 (Figure 4), which had a total of 195 measurements, including 29 independent HET visits. For periods less than about 1 year, we can exclude planets with $\mathrm{M} \sin i \gtrsim 2$ Neptune masses. Similar results were obtained for 16 Cyg B $(\mathrm{N}=161)$, where the limits approach a Neptune mass (Figure 6). Since the detection limits generally improve with the addition of more data and with higher-quality data, we can define a quantity to measure the goodness of the limits. A simple choice would be $N / \bar{\sigma}$, where $N$ is the total number of observations, and $\bar{\sigma}$ is the mean uncertainty of the radial-velocity measurements. The values of $N$ and $\bar{\sigma}$ are given in Table 2 .

In the HD 45350 system, the results of the dynamical simulations complement those of the detection limit determinations. Very tight limits are obtained in close orbits $(a \lesssim 0.2 \mathrm{AU})$. In this region, test particles were stable (Fig. 2) and our observations can exclude planets with M sin $i$ between about 1 and 4 Neptune masses. Similar results were obtained for the 16 Cyg B system, in which test particles remained stable inward of $a \sim 0.3 \mathrm{AU}$. In that region, planets of 1-3 Neptune masses can be excluded by our limits determinations (Fig. 6). In most of the limits determinations, there are multiple "blind spots" evident where the periodogram method failed to significantly recover the injected signals. Typically this occurs at certain trial periods for which the phase coverage of the observational data is poor, and often at the 1-month and 1-year windows.

For none of HD 37605 (Fig. 4), HD 80606 (Fig. 5), or HD 89744 (Fig. 6) could additional companions be ruled out below about $0.7 \mathrm{M}_{\mathrm{Jup}}$, and for most orbital periods tested, the limits were substantially worse. One possible explanation for this result is that the sampling of the observations was poorly distributed in phase for many of the injected test signals, making significant recovery by the periodogram method difficult. This is evidenced by the "jagged" regions in the plots. Also, the intrinsic scatter for those three systems was too large to permit tight limits determination. This is certainly reasonable for the F7 star HD 89744. The three systems with the best limits (HD 3651, HD 45350, and 16 Cyg B) also had the lowest rms scatter about their orbital solutions (mean=8.9 $\pm 1.4 \mathrm{~m} \mathrm{~s}^{-1}$; Table 1$)$. In contrast, the mean rms for the remaining three systems was $13.7 \pm 0.6 \mathrm{~m} \mathrm{~s}^{-1}$. Additional factors such as a paucity of data (HD 37605) and short time baselines (HD 80606, HD 89744) made the determination of useful companion limits challenging for some of the planetary systems in this study.

\section{SUMMARY}

We have shown that for a sample of six highly eccentric extrasolar planetary systems, there is no evidence for additional planets. Test particle simulations show that there are regions detectable by current surveys (i.e. for $a<2$ AU) where additional objects can exist. For HD 3651 and HD 37605, we find that protected resonances are also present. Combining these simulations with detection limits computed using new high-precision HET data combined with all available published data is particularly effective for the HD 3651 and HD 45350 systems. Additional short-period planets can be ruled out down to a few Neptune masses in the dynamically stable regions in these systems.

This material is based upon work supported by the National Aeronautics and Space Administration under Grant Nos. NNG04G141G and NNG05G107G issued through the Terrestrial Planet Finder Foundation Science program. We are grateful to the HET TAC for their generous allocation of telescope time for this project. We also would like to thank Barbara McArthur for her assistance with GaussFit software. We thank the referee, Greg Laughlin, for his careful review of this manuscript. This research has made use of NASA's Astrophysics Data System (ADS), and the SIMBAD database, operated at CDS, Strasbourg, France. The Hobby-Eberly Telescope (HET) is a joint project of the University of Texas at Austin, the Pennsylvania State University, Stanford University, Ludwig-Maximilians-Universität München, and Georg-August-Universität Göttingen The HET is named in honor of its principal benefactors, William P. Hobby and Robert E. Eberly. 


\section{REFERENCES}

Barnes, R., \& Raymond, S. N. 2004, ApJ, 617, 569

Butler, R. P., et al. 2006, ApJ, 646, 505

Cochran, W. D., et al. 2004, ApJ, 611, L133

Desidera, S., \& Barbieri, M. 2007, A\&A, 462, 345

Eggenberger, A., Udry, S., \& Mayor, M. 2004, A\&A, 417, 353

Endl, M., Cochran, W. D., Wittenmyer, R. A., \& Hatzes, A. P. 2006, AJ, 131, 3131

Fischer, D. A., Butler, R. P., Marcy, G. W., Vogt, S. S., \& Henry, G. W. 2003, ApJ, 590, 1081

Ford, E. B., Lystad, V., \& Rasio, F. A. 2005, Nature, 434, 873

Goldreich, P., \& Sari, R. 2003, ApJ, 585, 1024

Hauser, H. M., \& Marcy, G. W. 1999, PASP, 111, 321

Holman, M., Touma, J., \& Tremaine, S. 1997, Nature, 386, 254

Jefferys, W. H., Fitzpatrick, M. J., \& McArthur, B. E. 1987, Celestial Mechanics, 41, 39

Kozai, Y. 1962, AJ, 67, 591

Levison, H. F., \& Duncan, M. J. 1994, Icarus, 108, 18

Lin, D. N. C., \& Ida, S. 1997, ApJ, 477, 781

Lomb, N. R. 1976, Ap\&SS, 39, 447

Luhman, K. L., et al. 2007, ApJ, 654, 570

Malmberg, D., Davies, M. B., \& Chambers, J. E. 2007, MNRAS, L18

Mazeh, T., Krymolowski, Y., \& Rosenfeld, G. 1997, ApJ, 477, L103

McArthur, B. E., et al. 2004, ApJ, 614, L81

Menou, K., \& Tabachnik, S. 2003, ApJ, 583, 473

Mugrauer, M., Neuhäuser, R., Mazeh, T., Guenther, E., \& Fernández, M. 2004, Astronomische Nachrichten, 325, 718
Mugrauer, M., Seifahrt, A., Neuhäuser, R., \& Mazeh, T. 2006, MNRAS, 373, L31

Naef, D., et al. 2001, A\&A, 375, L27

Naef, D., et al. 2007, A\&A, in press, arXiv:0704.0917

Raghavan, D., Henry, T. J., Mason, B. D., Subasavage, J. P., Jao,

W.-C., Beaulieu, T. D., \& Hambly, N. C. 2006, ApJ, 646, 523

Rasio, F. A., \& Ford, E. B. 1996, Science, 274, 954

Rivera, E., \& Haghighipour, N. 2007, MNRAS, 374, 599

Rivera, E. J., et al. 2005, ApJ, 634, 625

Santos, N. C., et al. 2004, A\&A, 426, L19

Scargle, J. D. 1982, ApJ, 263, 835

Takeda, G., \& Rasio, F. A. 2005, ApJ, 627, 1001

Tull, R. G. 1998, Proc. SPIE, 3355, 387

Udry, S., et al. 2007, A\&A, submitted, arXiv:0704.3841

Vogt, S. S., Butler, R. P., Marcy, G. W., Fischer, D. A., Henry, G. W., Laughlin, G., Wright, J. T., \& Johnson, J. A. 2005, ApJ, 632, 638

Wilson, J. C., Kirkpatrick, J. D., Gizis, J. E., Skrutskie, M. F., Monet, D. G., \& Houck, J. R. 2001, AJ, 122, 1989

Wittenmyer, R. A., Endl, M., Cochran, W. D., Hatzes, A. P., Walker, G. A. H., Yang, S. L. S., \& Paulson, D. B. 2006, AJ, 132,177

Wittenmyer, R. A., Endl, M., \& Cochran, W. D. 2007, ApJ, 654, 625

Wu, Y., \& Murray, N. 2003, ApJ, 589, 605 
TABLE 1

Keplerian Orbital Solutions

\begin{tabular}{|c|c|c|c|c|c|c|c|c|}
\hline Planet & $\begin{array}{l}\text { Period } \\
\text { (days) }\end{array}$ & $\begin{array}{c}T_{0} \\
(\mathrm{JD}-2400000)\end{array}$ & $e$ & $\begin{array}{c}\omega \\
\text { (degrees) }\end{array}$ & $\begin{array}{c}\mathrm{K} \\
\left(\mathrm{m} \mathrm{s}^{-1}\right)\end{array}$ & $\begin{array}{l}\mathrm{M} \sin i \\
\left(\mathrm{M}_{\mathrm{Jup}}\right)\end{array}$ & $\begin{array}{c}a \\
(\mathrm{AU})\end{array}$ & $\begin{array}{c}\mathrm{rms} \\
\mathrm{m} \mathrm{s}^{-1}\end{array}$ \\
\hline HD $3651 \mathrm{~b}$ & $62.197 \pm 0.012$ & $53932.2 \pm 0.4$ & $0.630 \pm 0.046$ & $250.7 \pm 6.3$ & $15.6 \pm 1.1$ & $0.20 \pm 0.01$ & $0.280 \pm 0.006$ & 7.1 \\
\hline HD 37605 b & $55.027 \pm 0.009$ & $52992.8 \pm 0.1$ & $0.677 \pm 0.009$ & $218.4 \pm 1.7$ & $201.5 \pm 3.9$ & $2.39 \pm 0.12$ & $0.263 \pm 0.006$ & 13.0 \\
\hline HD $45350 \mathrm{~b}$ & $963.6 \pm 3.4$ & $51825.3 \pm 7.1$ & $0.778 \pm 0.009$ & $343.4 \pm 2.3$ & $58.0 \pm 1.7$ & $1.79 \pm 0.14$ & $1.92 \pm 0.07$ & 9.1 \\
\hline HD $80606 \mathrm{~b}$ & $111.428 \pm 0.002$ & $53421.928 \pm 0.004$ & $0.933 \pm 0.001$ & $300.4 \pm 0.3$ & $470.2 \pm 2.5$ & $4.10 \pm 0.12$ & $0.460 \pm 0.007$ & 13.5 \\
\hline HD $89744 \mathrm{~b}$ & $256.78 \pm 0.05$ & $53816.1 \pm 0.3$ & $0.689 \pm 0.006$ & $194.1 \pm 0.6$ & $263.2 \pm 3.9$ & $7.92 \pm 0.23$ & $0.91 \pm 0.01$ & 14.4 \\
\hline $16 \mathrm{Cyg} \mathrm{B} \mathrm{b}$ & $799.5 \pm 0.6$ & $50539.3 \pm 1.6$ & $0.689 \pm 0.011$ & $83.4 \pm 2.1$ & $51.2 \pm 1.1$ & $1.68 \pm 0.07$ & $1.68 \pm 0.03$ & 10.6 \\
\hline
\end{tabular}

TABLE 2

Summary of Radial-Velocity Data

\begin{tabular}{|c|c|c|c|c|}
\hline Star & $N$ & $\bar{\sigma}\left(\mathrm{m} \mathrm{s}^{-1}\right)$ & $\Delta T$ (days) & Source \\
\hline HD 3651 & 163 & 3.4 & & Butler et al. (2006) \\
\hline HD 3651 & 3 & 6.1 & & $\mathrm{HJS}^{\mathrm{a}}$ \\
\hline HD 3651 & 29 & 2.1 & & $\mathrm{HET}^{\mathrm{b}}$ \\
\hline HD 3651 (total) & 195 & 3.2 & 7083 & \\
\hline HD 37605 (total) & 43 & 2.9 & 1065 & HET \\
\hline HD 45350 & 38 & 2.8 & & Butler et al. (2006) \\
\hline HD 45350 & 28 & 4.2 & & HET \\
\hline HD 45350 & 47 & 8.9 & & HJS \\
\hline HD 45350 (total) & 113 & 5.7 & 2265 & \\
\hline HD 80606 & 61 & 13.7 & & Naef et al. (2001) \\
\hline HD 80606 & 46 & 5.1 & & Butler et al. (2006) \\
\hline HD 80606 & 23 & 2.5 & & HET \\
\hline HD 80606 (total) & 130 & 8.7 & 2893 & \\
\hline HD 89744 & 50 & 11.2 & & Butler et al. (2006) \\
\hline HD 89744 & 33 & 3.2 & & HET \\
\hline HD 89744 & 6 & 9.4 & & HJS \\
\hline HD 89744 (total) & 89 & 8.1 & 2687 & \\
\hline 16 Cyg B & 95 & 6.3 & & Butler et al. (2006) \\
\hline 16 Cyg B & 29 & 19.7 & & HJS Phase II $^{\mathrm{c}}$ \\
\hline 16 Cyg B & 37 & 7.4 & & HJS Phase III \\
\hline 16 Cyg B (total) & 161 & 9.0 & 6950 & \\
\hline
\end{tabular}

a McDonald Observatory $2.7 \mathrm{~m}$ Harlan J. Smith Telescope.

b McDonald Observatory $9.2 \mathrm{~m}$ Hobby-Eberly Telescope.

c Phase II indicates an earlier instrument setup detailed in Wittenmyer et al. (2006). Phase III is the current configuration. 

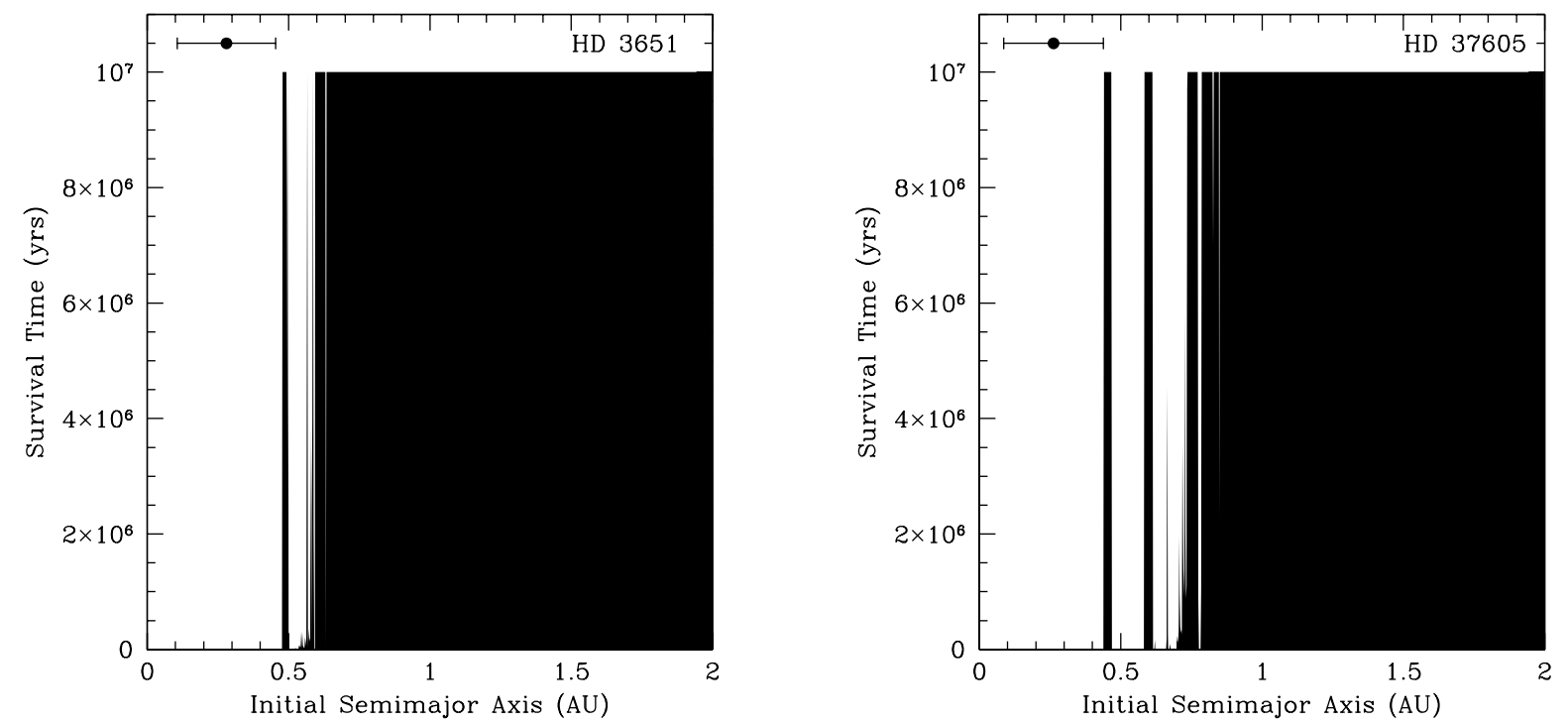

FIG. 1.- Left panel: Survival time as a function of initial semimajor axis for test particles in the HD 3651 system after $10^{7}$ yr. The filled regions indicate test particles which survived. The orbital excursion of HD 3561b is indicated by the horizontal error bars at the top. Particles were placed on initially circular orbits with $0.05<a<2.00$ AU. For all systems, the known planet removed particles which crossed its orbit. The dark region near 0.5 AU shows the stable 1:2 mean-motion resonance (MMR). Right panel: Same, but for the HD 37605 system. The dark regions near $0.45 \mathrm{AU}$ and 0.6 AU show the stable 1:2 and 1:3 MMRs.
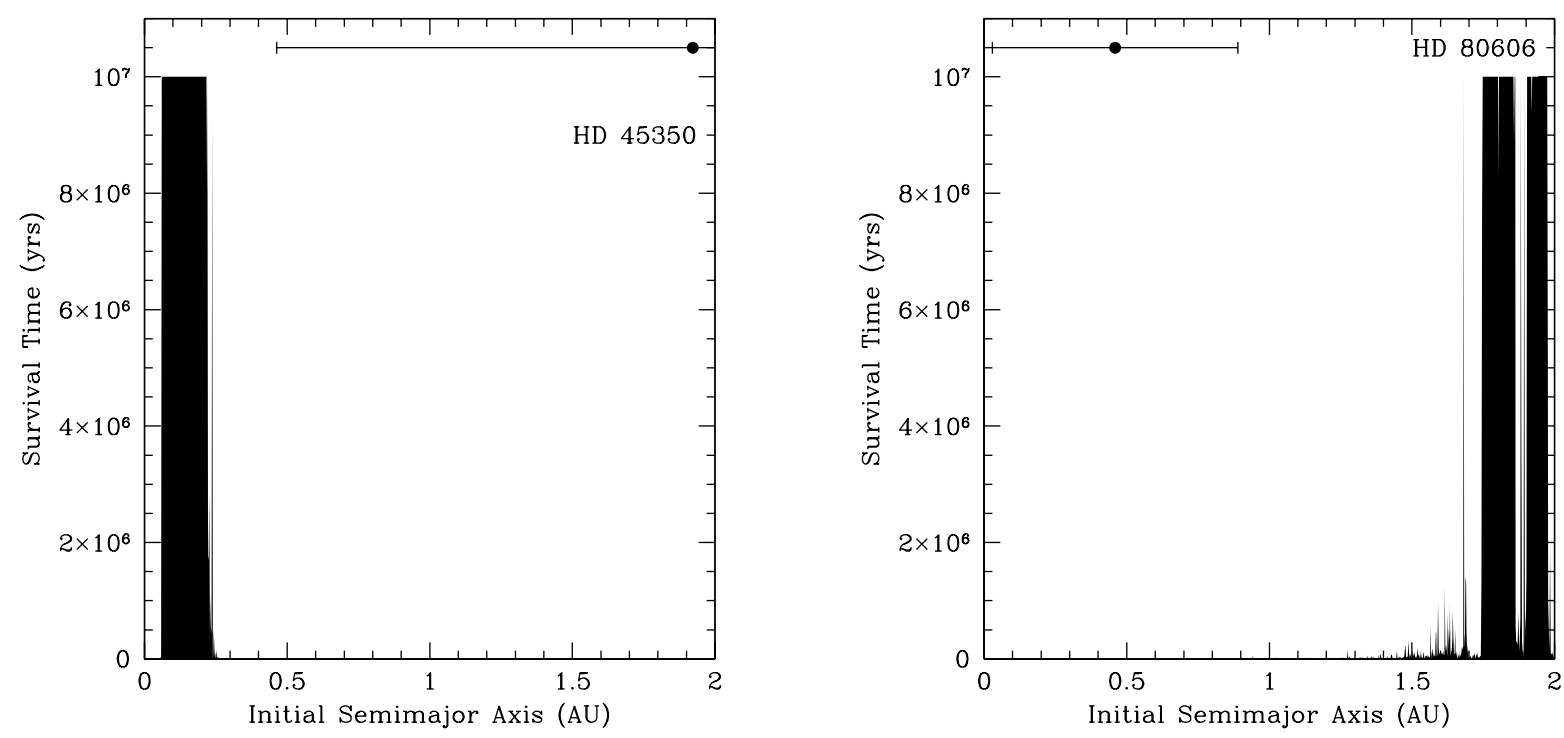

FIG. 2.- Same as Fig. 1, but for the HD 45350 (left) and HD 80606 (right) systems. 

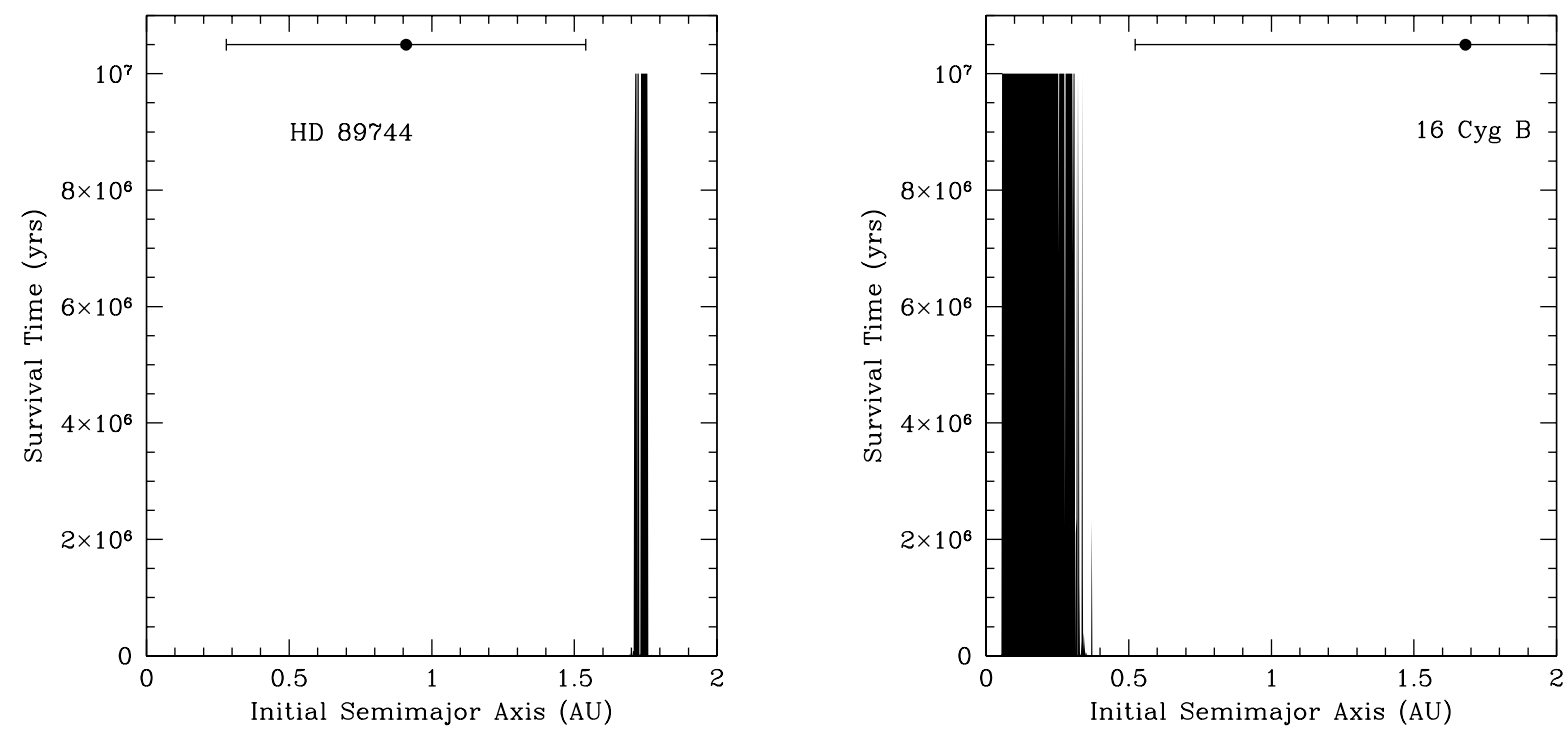

FIG. 3.- Same as Fig. 1, but for the HD 89744 (left) and 16 Cyg B (right) systems.
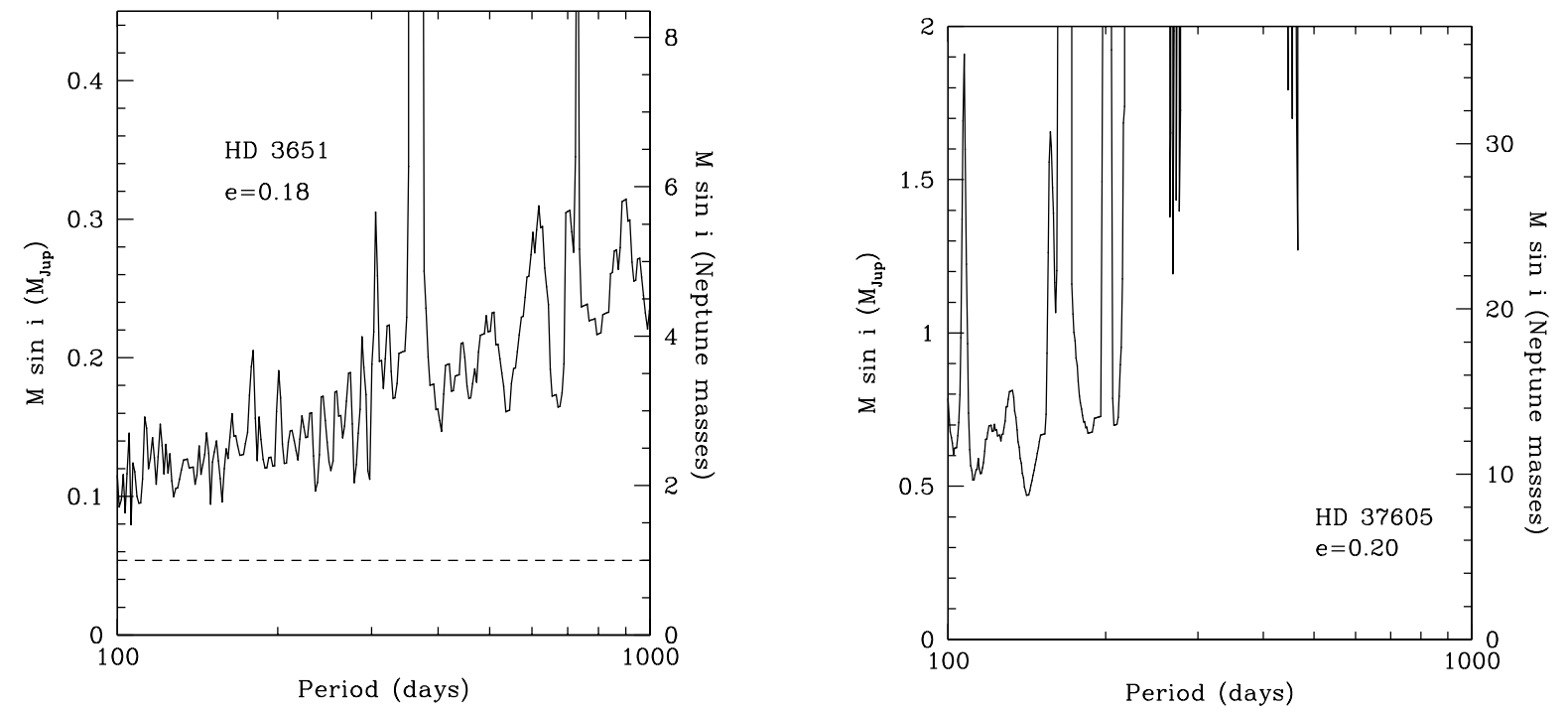

FIG. 4.- Left panel: Detection limits for additional planets in orbits with $e=0.18$ in the HD 3651 system. Planets in the parameter space above the plotted points are excluded at the $99 \%$ confidence level. The horizontal dashed line indicates the mass of Neptune. Right panel: Same, but for planets with $e=0.20$ in the HD 37605 system. 

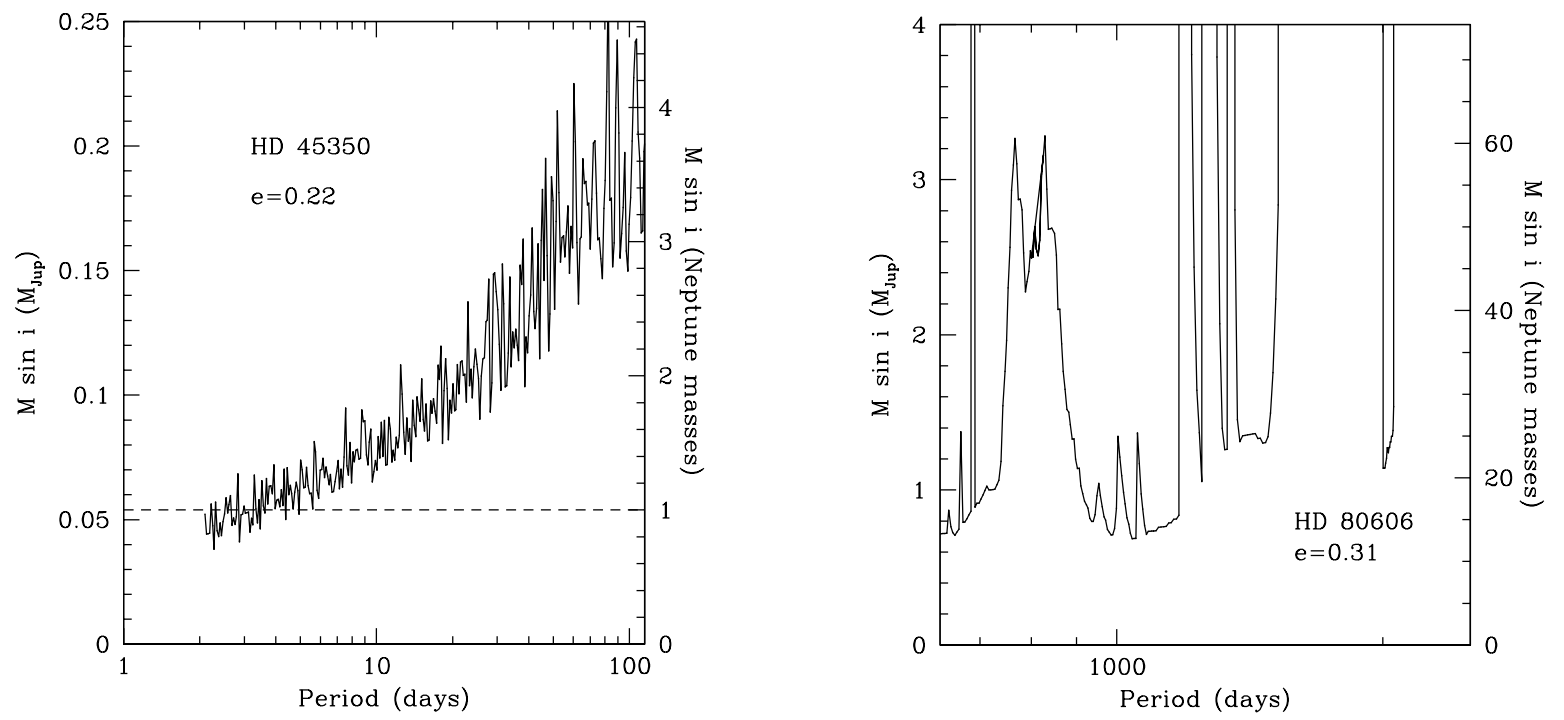

FIG. 5. - Left panel: Detection limits for additional planets with $e=0.22$ in the HD 45350 system. The horizontal dashed line indicates the mass of Neptune. Right panel: Same, but for planets with $e=0.31$ in the HD 80606 system. Planets in the parameter space above the plotted points are excluded at the $99 \%$ confidence level.
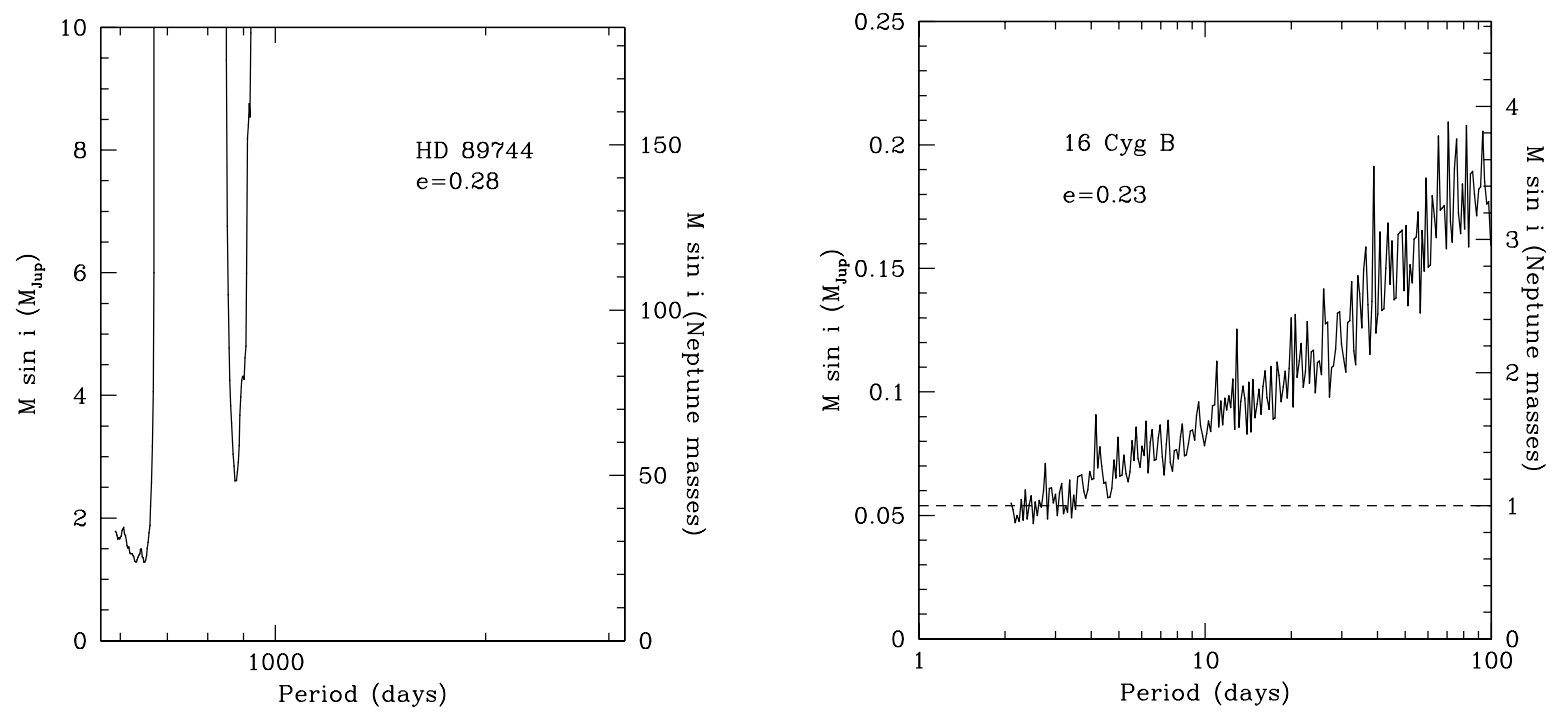

FIG. 6. - Left panel: Detection limits for additional planets with $e=0.28$ in the HD 89744 system. Right panel: Same, but for planets with $e=0.23$ in the 16 Cyg B system. The horizontal dashed line indicates the mass of Neptune. Planets in the parameter space above the plotted points are excluded at the $99 \%$ confidence level. 
TABLE 3

HET Radial Velocities For HD 3651

\begin{tabular}{|c|c|c|}
\hline JD-2400000 & Velocity $\left(\mathrm{m} \mathrm{s}^{-1}\right)$ & Uncertainty $\left(\mathrm{m} \mathrm{s}^{-1}\right)$ \\
\hline 53581.87326 & -19.1 & 2.9 \\
\hline 53581.87586 & -19.4 & 2.7 \\
\hline 53581.87846 & -20.7 & 2.7 \\
\hline 53600.79669 & -11.5 & 2.4 \\
\hline 53600.79860 & -15.5 & 3.0 \\
\hline 53600.80050 & -22.8 & 2.9 \\
\hline 53604.79166 & -15.8 & 1.9 \\
\hline 53604.79356 & -18.8 & 2.1 \\
\hline 53604.79548 & -21.3 & 2.1 \\
\hline 53606.78169 & -19.3 & 1.8 \\
\hline 53606.78360 & -14.8 & 2.1 \\
\hline 53606.78551 & -24.0 & 1.8 \\
\hline 53608.77236 & -18.8 & 1.9 \\
\hline 53608.77426 & -18.0 & 1.9 \\
\hline 53608.77617 & -18.8 & 1.8 \\
\hline 53615.96280 & -28.0 & 2.6 \\
\hline 53615.96471 & -31.9 & 2.4 \\
\hline 53615.96662 & -37.8 & 2.5 \\
\hline 53628.74050 & -6.8 & 2.2 \\
\hline 53628.74240 & -14.5 & 2.4 \\
\hline 53628.74431 & -5.5 & 2.2 \\
\hline 53669.61012 & -18.2 & 2.1 \\
\hline 53669.61203 & -19.2 & 2.2 \\
\hline 53669.61394 & -17.7 & 2.4 \\
\hline 53678.78954 & -10.6 & 2.4 \\
\hline 53678.79141 & -8.6 & 2.3 \\
\hline 53678.79332 & -2.3 & 2.1 \\
\hline 53682.78423 & -15.4 & 2.2 \\
\hline 53682.78609 & -15.0 & 2.3 \\
\hline 53682.78801 & -11.9 & 2.3 \\
\hline 53687.77684 & 11.3 & 2.2 \\
\hline 53687.77875 & 8.7 & 2.2 \\
\hline 53687.78066 & 15.9 & 2.2 \\
\hline 53691.75967 & 9.6 & 2.2 \\
\hline 53691.76158 & 20.3 & 2.1 \\
\hline 53691.76349 & 15.9 & 2.0 \\
\hline 53696.75837 & 16.1 & 1.8 \\
\hline 53696.76028 & 18.6 & 1.8 \\
\hline 53696.76220 & 20.0 & 2.0 \\
\hline 53694.75275 & 18.0 & 1.9 \\
\hline 53694.75466 & 15.1 & 2.0 \\
\hline 53694.75656 & 17.8 & 2.0 \\
\hline 53955.83401 & -0.5 & 1.9 \\
\hline 53955.83593 & -1.2 & 2.0 \\
\hline 53955.83785 & 1.3 & 1.9 \\
\hline 53956.82850 & 0.4 & 2.0 \\
\hline 53956.83046 & -1.0 & 2.0 \\
\hline 53956.83236 & -5.4 & 2.2 \\
\hline 53957.82201 & -2.1 & 2.0 \\
\hline 53957.82392 & -1.3 & 2.0 \\
\hline 53957.82583 & -3.6 & 2.0 \\
\hline 53973.80721 & 9.8 & 7.3 \\
\hline 53973.81020 & 3.5 & 2.3 \\
\hline 53973.81200 & -3.5 & 2.0 \\
\hline 53976.78393 & -10.4 & 2.4 \\
\hline 53976.78586 & -5.4 & 2.1 \\
\hline 53976.78778 & -6.7 & 2.3 \\
\hline 53978.97197 & -3.8 & 2.6 \\
\hline 53985.95886 & -9.0 & 2.3 \\
\hline 53985.96079 & 4.3 & 3.3 \\
\hline 53987.95335 & -8.3 & 2.2 \\
\hline 53987.95527 & -8.0 & 2.2 \\
\hline 53987.95719 & -12.0 & 2.3 \\
\hline 53989.73817 & -13.2 & 2.2 \\
\hline 53989.74009 & -13.2 & 2.1 \\
\hline 53989.74203 & -18.6 & 2.1 \\
\hline 54003.70719 & 2.0 & 2.2 \\
\hline 54003.70915 & 4.7 & 2.4 \\
\hline 54005.68297 & 7.0 & 2.5 \\
\hline 54005.68488 & 11.1 & 2.0 \\
\hline 54005.68690 & 10.2 & 2.1 \\
\hline 54056.77919 & -7.5 & 2.2 \\
\hline 54056.78110 & -11.5 & 2.1 \\
\hline 54056.78302 & -9.6 & 2.3 \\
\hline 54062.55119 & 20.1 & 1.8 \\
\hline 54062.55312 & 21.9 & 2.0 \\
\hline 54062.55505 & 20.9 & 2.0 \\
\hline 54064.54710 & 12.8 & 2.0 \\
\hline 54064.54902 & 16.7 & 2.1 \\
\hline 54064.55094 & 16.6 & 2.1 \\
\hline 54130.55316 & 19.1 & 2.4 \\
\hline
\end{tabular}


TABLE 4

HET Radial Velocities FOR HD 37605

\begin{tabular}{rrr}
\hline \hline JD-2400000 & Velocity $\left(\mathrm{m} \mathrm{s}^{-1}\right)$ & Uncertainty $\left(\mathrm{m} \mathrm{s}^{-1}\right)$ \\
\hline 53002.67151 & 487.6 & 3.8 \\
53003.68525 & 495.5 & 3.0 \\
53006.66205 & 496.2 & 3.0 \\
53008.66407 & 501.3 & 2.9 \\
53010.80477 & 499.8 & 2.9 \\
53013.79399 & 482.1 & 2.6 \\
53042.72797 & 269.7 & 2.8 \\
53061.66756 & 489.0 & 2.6 \\
53065.64684 & 479.0 & 2.8 \\
53071.64383 & 463.8 & 2.6 \\
53073.63819 & 460.4 & 2.6 \\
53082.62372 & 422.8 & 2.5 \\
53083.59536 & 422.2 & 2.8 \\
53088.59378 & 418.6 & 4.0 \\
53089.59576 & 379.1 & 2.2 \\
53092.59799 & 343.7 & 2.5 \\
53094.58658 & 323.2 & 2.4 \\
53095.58642 & 302.1 & 2.4 \\
53096.58744 & 302.1 & 3.2 \\
53098.57625 & 193.8 & 2.7 \\
53264.95137 & 164.9 & 3.0 \\
53265.94744 & 112.9 & 3.0 \\
53266.94598 & 113.2 & 3.7 \\
53266.95948 & 74.6 & 3.6 \\
53266.97396 & 119.2 & 8.0 \\
53283.92241 & 471.6 & 2.7 \\
53318.81927 & 213.3 & 3.0 \\
53335.92181 & 496.9 & 2.6 \\
53338.90602 & 493.9 & 2.6 \\
53377.81941 & 109.1 & 2.7 \\
53378.81189 & 214.6 & 2.7 \\
53379.80225 & 338.3 & 2.6 \\
53381.64429 & 436.1 & 2.7 \\
53384.64654 & 482.9 & 2.8 \\
53724.85584 & 468.2 & 2.7 \\
53731.69723 & 435.4 & 2.6 \\
53738.67472 & 404.3 & 2.6 \\
53743.81020 & 400.5 & 3.1 \\
53748.64724 & 348.4 & 2.7 \\
54039.85015 & 272.5 & 2.9 \\
54054.96457 & 437.4 & \\
54055.95279 & 422.0 & \\
54067.76282 & 376.4 & \\
& & \\
\hline & & \\
& & \\
& &
\end{tabular}


TABLE 5

HET Radial Velocities for HD 80606

\begin{tabular}{rrr}
\hline \hline JD-2400000 & Velocity $\left(\mathrm{m} \mathrm{s}^{-1}\right)$ & Uncertainty $\left(\mathrm{m} \mathrm{s}^{-1}\right)$ \\
\hline 53346.88103 & -20.8 & 3.0 \\
53358.02089 & -49.5 & 2.7 \\
53359.82400 & -60.4 & 3.0 \\
53361.02985 & -64.7 & 2.5 \\
53365.03079 & -77.4 & 2.4 \\
53373.98282 & -88.4 & 3.0 \\
53377.80112 & -105.5 & 2.4 \\
53379.75230 & -109.3 & 2.7 \\
53389.74170 & -115.3 & 2.5 \\
53391.74400 & -129.4 & 2.4 \\
53395.72763 & -146.4 & 2.3 \\
53399.72518 & -158.4 & 2.5 \\
53401.72497 & -174.7 & 2.7 \\
53414.67819 & -219.8 & 3.0 \\
53421.85529 & 261.0 & 2.2 \\
53423.86650 & 322.1 & 2.0 \\
53424.85231 & 245.9 & 2.1 \\
53432.87120 & 87.5 & 1.9 \\
53433.60628 & 70.0 & 2.1 \\
53446.79322 & 4.5 & 1.9 \\
54161.85400 & -109.5 & 2.8 \\
54166.83797 & -119.3 & 2.4 \\
54186.76189 & -184.2 & 2.3 \\
& & \\
\hline
\end{tabular}


TABLE 6

HET Radial Velocities For HD 89744

\begin{tabular}{|c|c|c|}
\hline JD-2400000 & Velocity $\left(\mathrm{m} \mathrm{s}^{-1}\right)$ & Uncertainty $\left(\mathrm{m} \mathrm{s}^{-1}\right)$ \\
\hline 53709.89685 & -184.5 & 2.3 \\
\hline 53723.85188 & -238.6 & 2.2 \\
\hline 53723.85367 & -238.2 & 2.5 \\
\hline 53723.85546 & -227.7 & 2.3 \\
\hline 53727.84394 & -238.9 & 2.5 \\
\hline 53727.84573 & -244.9 & 2.4 \\
\hline 53727.84752 & -242.9 & 2.6 \\
\hline 53736.81887 & -257.6 & 2.5 \\
\hline 53736.82100 & -248.2 & 2.9 \\
\hline 53736.82315 & -253.4 & 2.4 \\
\hline 53738.03261 & -246.7 & 2.8 \\
\hline 53738.03441 & -243.3 & 2.4 \\
\hline 53738.03620 & -236.0 & 2.5 \\
\hline 53738.80860 & -240.5 & 2.6 \\
\hline 53738.81040 & -258.9 & 2.4 \\
\hline 53738.81219 & -249.3 & 2.5 \\
\hline 53734.81795 & -242.8 & 2.6 \\
\hline 53734.81973 & -243.9 & 2.8 \\
\hline 53734.82152 & -248.5 & 2.4 \\
\hline 53742.79119 & -252.0 & 2.8 \\
\hline 53742.79299 & -257.2 & 2.8 \\
\hline 53742.79479 & -239.7 & 2.8 \\
\hline 53751.78199 & -257.4 & 2.9 \\
\hline 53751.78378 & -263.1 & 2.5 \\
\hline 53751.78558 & -268.0 & 2.3 \\
\hline 53753.78155 & -273.1 & 2.5 \\
\hline 53753.78381 & -278.7 & 2.5 \\
\hline 53753.78607 & -266.4 & 2.4 \\
\hline 53755.76038 & -286.6 & 2.3 \\
\hline 53755.76218 & -266.5 & 2.6 \\
\hline 53755.76397 & -274.9 & 2.7 \\
\hline 53746.81506 & -257.1 & 1.9 \\
\hline 53746.81778 & -250.9 & 2.1 \\
\hline 53746.82051 & -245.2 & 2.3 \\
\hline 53757.77002 & -277.6 & 2.4 \\
\hline 53757.77181 & -280.3 & 2.4 \\
\hline 53757.77360 & -288.7 & 2.2 \\
\hline 53797.64609 & -439.8 & 3.1 \\
\hline 53797.64834 & -462.6 & 2.8 \\
\hline 53797.65059 & -452.5 & 2.9 \\
\hline 53809.62428 & -658.6 & 2.4 \\
\hline 53809.62700 & -658.8 & 2.5 \\
\hline 53809.62972 & -659.2 & 2.3 \\
\hline 53837.76359 & -304.3 & 3.0 \\
\hline 53837.76670 & -324.0 & 2.9 \\
\hline 53837.78731 & -308.6 & 2.7 \\
\hline 53837.79077 & -285.2 & 2.6 \\
\hline 53866.69987 & -215.9 & 1.7 \\
\hline 53866.70329 & -228.3 & 1.7 \\
\hline 53866.70670 & -220.4 & 1.8 \\
\hline 53868.68349 & -251.6 & 3.8 \\
\hline 53868.68562 & -208.6 & 2.9 \\
\hline 53868.68777 & -247.4 & 9.7 \\
\hline 53875.66956 & -215.7 & 1.6 \\
\hline 53883.65565 & -213.8 & 1.8 \\
\hline 53883.65837 & -209.2 & 1.7 \\
\hline 53883.66109 & -200.4 & 1.7 \\
\hline 53890.63776 & -203.4 & 1.7 \\
\hline 53890.63954 & -202.6 & 1.9 \\
\hline 53890.64134 & -203.2 & 1.9 \\
\hline 53893.62959 & -193.8 & 2.0 \\
\hline 53893.63139 & -189.3 & 1.9 \\
\hline 53893.63318 & -189.7 & 1.8 \\
\hline 54047.94811 & -375.2 & 4.8 \\
\hline 54047.94991 & -353.2 & 4.5 \\
\hline 54047.95172 & -362.6 & 4.4 \\
\hline 54050.96248 & -415.0 & 2.6 \\
\hline 54050.96453 & -423.0 & 2.5 \\
\hline 54050.96657 & -420.1 & 2.4 \\
\hline 54052.96488 & -426.8 & 2.3 \\
\hline 54052.96762 & -437.1 & 2.5 \\
\hline 54052.97035 & -447.6 & 2.5 \\
\hline 54056.94606 & -468.0 & 3.0 \\
\hline 54056.94786 & -466.4 & 2.6 \\
\hline 54056.94964 & -479.4 & 2.8 \\
\hline 54063.92981 & -599.1 & 2.1 \\
\hline 54063.93166 & -594.8 & 2.3 \\
\hline 54063.93348 & -592.3 & 2.4 \\
\hline 54073.91213 & -685.8 & 2.8 \\
\hline 54073.91476 & -688.7 & 2.9 \\
\hline 54073.91739 & -704.4 & 2.7 \\
\hline
\end{tabular}

